\title{
HIP 21539 is not a past very close neighbour of the Sun
}

\author{
F. Crifo ${ }^{1}$, C. Soubiran ${ }^{2}$, G. Jasniewicz ${ }^{3}$, D. Katz ${ }^{1}$, P. Sartoretti ${ }^{1}$, and P. Panuzzo ${ }^{1}$ \\ ${ }^{1}$ GEPI, Observatoire de Paris, PSL Research University, CNRS, Univ. Paris Diderot, Sorbonne Paris Cité, 5 place Jules Janssen, \\ 92190 Meudon, France \\ e-mail: francoise.crifo@obspm.fr \\ ${ }^{2}$ Laboratoire d'astrophysique de Bordeaux, Univ. Bordeaux, CNRS, B18N, allée Geoffroy Saint-Hilaire, 33615 Pessac, France \\ 3 LUPM, UMR CNRS/Université de Montpellier, CC 72, Université de Montpellier, 34095 Montpellier Cedex 05, France
}

Received 30 January 2017 / Accepted 1 March 2017

\begin{abstract}
Aims. A previous study claimed that the star HIP 21539 passed close to the Sun, at a distance of 1.9 pc, around 0.14 Myr ago. We show that this is not the case.

Methods. We redetermined the trajectory of the star relative to the Sun using a new accurate radial velocity from the HARPS spectrograph combined with the recent Gaia-TGAS astrometry.

Results. With this new data, the closest approach of HIP 21539 to the Sun is now 17 pc, instead of 1.9 pc.

Conclusions. At this distance, the star has not perturbed the Oort cloud.
\end{abstract}

Key words. solar neighborhood - techniques: radial velocities - catalogs - stars: kinematics and dynamics

\section{Introduction}

We present a short improvement to the interesting paper by Dybczyński \& Berski (2015) about close stellar passages at less than 2 pc from the Sun with a possible impact on cometary orbits. As part of the work aimed at defining stellar radial velocity standards (RV-STD) for the calibration of the Gaia Radial Velocity Spectrometer (RVS; Crifo et al. 2010; Soubiran et al. 2013), we obtained a much better radial velocity (RV) for one of their targets, i.e. HIP 21539. This new RV is derived from three independent observations by the HARPS spectrograph and rules out the possibility for this star to have had such a close passage in the recent past. New values for the date and minimum distance are estimated with the straight line approximation.

\section{The problem}

Dybczyński \& Berski (2015) carefully examined the possible candidates for close passages of already nearby stars at a distance less than 2 pc from the Sun, as such objects may strongly perturb the Oort cloud. Just before, Bailer-Jones (2015) carried out a very similar work. Both papers used the XHIP catalogue by Anderson \& Francis (2012) as an entrance list. The XHIP catalogue contains all necessary data. Parallaxes and proper motions are taken from the HIP-2 catalogue (van Leeuwen 2007): but radial velocities come from a vast compilation made by Anderson and Francis, who really searched deep in the literature for all possible existing data.

The star HIP 21539 is found only in the paper by Dybczyński \& Berski (2015); it is supposed to have had its closest approach $0.14 \mathrm{Myr}$ ago at a perihelion distance of $1.92 \mathrm{pc}$. The corresponding radial velocity is $248 \mathrm{~km} \mathrm{~s}^{-1}$, issued from the Barbier-Brossat et al. (1994) catalogue, itself referring to Contreras \& Stock (1970), "Radial velocities for twenty-three stars selected from an objective prism survey are communicated.
The data indicate that the peculiar G- and K-stars included in the program constitute a high velocity group". The data quality is quoted as "D" in XHIP, i.e. the lowest quality. No other value is available in Simbad or Vizier.

\section{New radial velocity}

In order to find additional RV-STD for the Gaia RVS, we searched the AMBRE-HARPS catalogue (De Pascale et al. 2014). This catalogue provides atmospheric parameters for the ESO:HARPS archived spectra, together with radial velocities either derived by the ESO:HARPS reduction pipeline or by the AMBRE pipeline. The HARPS spectrograph is a velocimeter mounted on the ESO $3.6 \mathrm{~m}$ telescope at La Silla, with a resolving power of $R=\lambda / \Delta \lambda=115000$; for more details see Pepe et al. (2000).

To be consistent with our previous lists of RV-STD candidates (Crifo et al. 2010; Soubiran et al. 2013), the RV measurements must be expressed in the SOPHIE scale; SOPHIE is another velocimeter at Observatoire de Haute-Provence with $R=75000$ and a reduction pipeline similar to that of HARPS.

For HIP 21539, the AMBRE-HARPS catalogue provides three measurements of RV from the ESO pipeline at dates $2003 / 12 / 11,2004 / 02 / 01$, and 2004/11/26, i.e. a time span of 351 days.

The weighted average of these three values gives

$\mathrm{RV}=26.926 \mathrm{~km} \mathrm{~s}^{-1} ; \sigma_{\mathrm{RV}}=0.0026 \mathrm{~km} \mathrm{~s}^{-1}$.

The star was integrated into the list of radial velocity standards for the RVS.

\section{Linear approximation}

We now calculate a new approximate minimum distance and corresponding date for the closest approach of the star to 


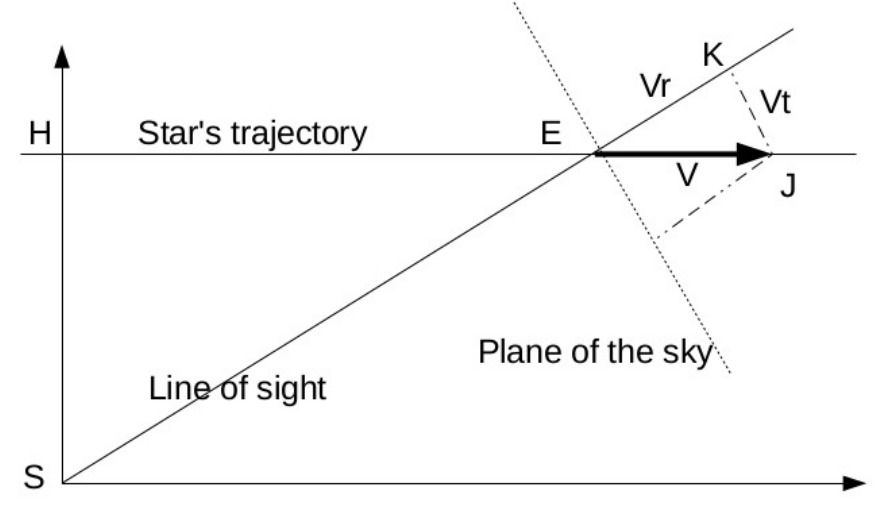

Fig. 1. Trajectory of the star relative to the Sun. The star E moves uniformly along the straight line. $\mathrm{H}$ is the perihelion.

the Sun, called $d_{\mathrm{ph}}$ and $t_{\mathrm{ph}} \quad$ (distance and time from perihelion) according to Bailer-Jones (2015). Bailer-Jones and Dybczyński \& Berski first computed $d_{\mathrm{ph}}$ and $t_{\mathrm{ph}}$ with the linear approximation and then with the introduction of the Galactic potential perturbing the linear motion; Bailer-Jones used the linear approximation for a first gross selection within the XHIP catalogue (Anderson \& Francis 2012). In their Table 2, Dybczyński \& Berski (2015) compare the results of the two calculations: the difference for $d_{\mathrm{ph}}$ becomes noticeable for $\left|t_{\mathrm{ph}}\right|>3 \mathrm{Myr}$, i.e. for far-away stars that travelled long enough in time to have felt the influence of the Galactic potential. In our case, the linear approximation is largely sufficient; moreover the final value of $t_{\mathrm{ph}}(\sim 1 \mathrm{Myr})$ shows that it is appropriate.

Figure 1, taken from Green (1985, Fig. 11.1), illustrates the positions: $\mathrm{S}=\mathrm{Sun} ; \mathrm{E}$ is the star at our epoch $(\mathrm{SE}=r)$, and assumed to move with an uniform velocity along a straight line; $\mathrm{H}$ is the closest approach to the Sun (perihelion for Bailer-Jones), at a distance $\mathrm{SH}=d_{\mathrm{ph}}$ and a time $t_{\mathrm{ph}}$ to be calculated (origin of time at $\mathrm{E}$ ). The total velocity $\boldsymbol{V}$ is projected over the line-of-sight $\mathrm{SE}$ and the plane of the sky; the components are $V_{r}$ and $V_{t}$.

In the right triangle EKJ we have $\mathrm{EK}=V_{r} ; \mathrm{JK}=V_{t}$. By comparing the right triangles EKJ and EHS, we may write

$\mathrm{SH} / \mathrm{SE}=\mathrm{JK} / \mathrm{JE}=V_{t} / V_{\text {tot }}=d_{\mathrm{ph}} / r$

$\mathrm{HE} / \mathrm{SE}=t_{\mathrm{ph}} \cdot V_{\text {tot }} / r=\mathrm{KE} / \mathrm{JE}=V_{r} / V_{\text {tot }}$.

Hence,

$d_{\mathrm{ph}}=r \cdot V_{t} / V_{\mathrm{tot}} ; t_{\mathrm{ph}}=-r \cdot V_{r} / V_{\mathrm{tot}}^{2}$.

A sign "-" must be introduced in front of the expression of $t_{\mathrm{ph}}$, as the origin of time is supposed to be at position $\mathrm{E}$, and $V_{r}$ is positive when the star is receding from the Sun.

The values $V_{t}$ and $V_{\text {tot }}$ are calculated from the proper motion components $\mu_{\alpha}$ and $\mu_{\delta}$ and the parallax $\varpi$,

$V_{t}=k \cdot \sqrt{\mu_{\alpha}^{2}+\mu_{\delta}^{2}} / \varpi ; \quad V_{\mathrm{tot}}=\sqrt{V_{t}^{2}+V_{r}^{2}}$,

where $k=4.74$ is the coefficient converting the $\operatorname{arcsec} \mathrm{yr}^{-1}$ in $\mathrm{km} \mathrm{s}^{-1}$ (see Bailer-Jones Eq. (5); Green, Eq. (11.8)).

\section{Results}

The resulting numerical values are given in Table 1 in the following three cases:

- cases "old" and "new" for the two values of RV: the old bad value and the new HARPS value, combined with
Table 1. Calculation of old and new distance of perihelion.

\begin{tabular}{lrrrrrr}
\hline \hline Case & $\begin{array}{r}\varpi \\
\mathrm{mas}\end{array}$ & $\begin{array}{r}\mu_{\alpha} \\
\mathrm{mas} \mathrm{yr}^{-1}\end{array}$ & $\begin{array}{r}\mu_{\delta} \\
\mathrm{mas} \mathrm{yr}^{-1}\end{array}$ & $\begin{array}{r}V_{r} \\
\mathrm{~km} \mathrm{~s}^{-1}\end{array}$ & $\begin{array}{r}d_{\mathrm{ph}} \\
\mathrm{pc}\end{array}$ & $\begin{array}{r}t_{\mathrm{ph}} \\
\mathrm{Myr}\end{array}$ \\
\hline Old & 28.580 & -80.73 & 15.70 & 248.000 & 1.92 & -0.14 \\
Sig & 1.340 & 1.08 & 1.28 & 20.000 & 0.20 & 0.04 \\
& & & & & & \\
New & 28.580 & -80.73 & 15.70 & 26.926 & 15.81 & -1.03 \\
Sig & 1.340 & 1.08 & 1.28 & 0.003 & 1.06 & 0.05 \\
& & & & & & \\
TGAS & 27.270 & -81.31 & 15.27 & 26.926 & 17.27 & -1.06 \\
Sig & 0.280 & 0.08 & 0.09 & 0.003 & 0.25 & 0.01 \\
\hline
\end{tabular}

the parallax and proper motion from HIPPARCos-2, as in Dybczyński \& Berski, Table 2;

- case "TGAS" for the HARPS RV combined with the recently published parallax and proper motion from the TGAS Catalogue: Tycho-Gaia subset, available at CDS (Gaia Collaboration 2016).

In Table 1, each "case" is made of two lines: the upper line contains the data itself; the lower line (noted "sig") contains the corresponding errors, taken from SIMBAD (HIP2) or TGAS. An arbitrary error of $20 \mathrm{~km} \mathrm{~s}^{-1}$ was adopted for the old RV of $248 \mathrm{~km} \mathrm{~s}^{-1}$ (order of magnitude for error on radial velocities obtained with objective prism, but unrealistic here). Using the TGAS data instead of HIP-2 improves the accuracy of $d_{\mathrm{ph}}$ and $t_{\mathrm{ph}}$.

With updated RV, parallax and proper motion, the closest approach of HIP 21539 to the Sun is now $17.3 \mathrm{pc}, \sim 1 \mathrm{Myr}$ ago instead of $1.9 \mathrm{pc}$ and $0.14 \mathrm{Myr}$ ago, as first computed by Dybczyński \& Berski.

\section{Conclusions}

These new data show that HIP 21539 did not pass very close to the Sun; it therefore certainly did not perturb the Oort cloud. This short paper shows the importance of reliable RV for a good description of the solar neighbourhood and Galactic mechanics. The RVS on board Gaia is expected to provide radial velocities for more than 100 millions stars and revolutionize our knowledge of kinematics in the solar neighbourhood.

Acknowledgements. Many thanks to the CDS, particularly for the SIMBAD database and the Vizier section with so many old catalogues and data that are carefully stored and made available. This work has made use (for Table 1) of data from the European Space Agency (ESA) mission Gaia (http://www . cosmos.esa.int/gaia), processed by the Gaia Data Processing and Analysis Consortium (DPAC, http://www.cosmos.esa.int/web/gaia/dpac/ consortium). Funding for the DPAC has been provided by national institutions, in particular the institutions participating in the Gaia Multilateral Agreement.

\section{References}

Anderson, E., \& Francis, C. 2012, Astron. Lett., 38, 331

Bailer-Jones, C. A. L. 2015, A\&A, 575, A35

Barbier-Brossat, M., Petit, M., \& Figon, P. 1994, A\&AS, 108, 603

Contreras, C., \& Stock, J. 1970, Publications of the Department of Astronomy

University of Chile, 2, 40

Crifo, F., Jasniewicz, G., Soubiran, C., et al. 2010, A\&A, 524, A10

De Pascale, M., Worley, C. C., de Laverny, P., et al. 2014, A\&A, 570, A68

Dybczyński, P. A., \& Berski, F. 2015, MNRAS, 449, 2459

Gaia Collaboration (Brown, A. G. A., et al.) 2016, A\&A, 595, A2

Green, R. M. 1985, Spherical astronomy (Cambridge, New York: Cambridge University Press)

Pepe, F., Mayor, M., Delabre, B., et al. 2000, in SPIE Conf. Ser. 4008, eds. M. Iye, \& A. F. Moorwood, 582

Soubiran, C., Jasniewicz, G., Chemin, L., et al. 2013, A\&A, 552, A64

van Leeuwen, F. 2007, A\&A, 474, 653 